\title{
Integrated computational approach to the analysis of RNA-seq data reveals new transcriptional regulators of psoriasis
}

\author{
Alena Zolotarenko ${ }^{1}$, Evgeny Chekalin ${ }^{1}$, Alexandre Mesentsev ${ }^{1}$, Ludmila Kiseleva ${ }^{2}$, Elena Gribanova ${ }^{2}$, \\ Rohini Mehta ${ }^{3}$, Ancha Baranova ${ }^{3,4,5,6}$, Tatiana V Tatarinova ${ }^{6,7,8}$, Eleonora S Piruzian ${ }^{1}$ and Sergey Bruskin ${ }^{1,5}$ \\ Psoriasis is a common inflammatory skin disease with complex etiology and chronic progression. To provide novel insights into \\ the regulatory molecular mechanisms of the disease, we performed RNA sequencing analysis of 14 pairs of skin samples \\ collected from patients with psoriasis. Subsequent pathway analysis and extraction of the transcriptional regulators governing \\ psoriasis-associated pathways was executed using a combination of the MetaCore Interactome enrichment tool and the \\ cisExpress algorithm, followed by comparison to a set of previously described psoriasis response elements. A comparative \\ approach allowed us to identify $\mathbf{4 2}$ core transcriptional regulators of the disease associated with inflammation (NFkB, IRF9, \\ JUN, FOS, SRF), the activity of T cells in psoriatic lesions (STAT6, FOXP3, NFATC2, GATA3, TCF7, RUNX1), the hyper- \\ proliferation and migration of keratinocytes (JUN, FOS, NFIB, TFAP2A, TFAP2C) and lipid metabolism (TFAP2, RARA, VDR). In \\ addition to the core regulators, we identified 38 transcription factors previously not associated with the disease that can clarify \\ the pathogenesis of psoriasis. To illustrate these findings, we analyzed the regulatory role of one of the identified transcription \\ factors (TFs), FOXA1. Using ChIP-seq and RNA-seq data, we concluded that the atypical expression of the FOXA1 TF is an \\ important player in the disease as it inhibits the maturation of naive T cells into the $(C D 4+F O X A 1+C D 47+C D 69+P D-L 1$ (hi) \\ FOXP3-) regulatory T cell subpopulation, therefore contributing to the development of psoriatic skin lesions.
}

Experimental \& Molecular Medicine (2016) 48, e268; doi:10.1038/emm.2016.97; published online 4 November 2016

\section{INTRODUCTION}

Psoriasis is a common chronic immune-mediated inflammatory condition characterized by complex alterations of cell signaling leading to the progression of the disease. The observed synergy between the aberrant activation of immune cells and the abnormal proliferation and differentiation of keratinocytes leads to the development of typical psoriatic symptoms-red scaly thickened plaques on the skin surface. Another feature of psoriasis is a 'cytokine storm' that begins locally within the skin and then spreads throughout the body in form of systemic inflammation that contributes to the development of comorbidities, such as heart disease, stroke, diabetes and psoriatic arthritis.

To identify the key signaling cascades and gene expression alterations causing disease development and progression, we performed RNA-sequencing (RNA-seq) analysis of the skin transcriptome in 14 patients with psoriasis. Compared with other methods of gene expression analysis, RNA-seq provides a more precise measurement of transcription levels, a wider dynamic range of detection and more reproducible results. It was noted in Quigley ${ }^{1}$ that while for the most abundant transcripts both microarray and RNA-seq produce similar results, RNA-seq is capable of identifying a large number of transcripts expressed at low levels that could not be confidently called as differentially expressed when using microarrays to analyze the same number of samples.

In this study, we present the results of RNA-seq analysis that allowed us to identify important signaling cascades enriched with differentially expressed genes (DEGs) and highlight potential transcriptional regulators contributing to

\footnotetext{
${ }^{1}$ Laboratory of Functional Genomics, Vavilov Institute of General Genetics, Moscow, Russian Federation; ${ }^{2}$ Bryansk Regional STD and Dermatology Center, Bryansk Region, Russian Federation; ${ }^{3}$ School of Systems Biology, George Mason University, Fairfax, Virginia, USA; ${ }^{4}$ Russian Centre for Medical Genetics RAMN, Moscow, Russian Federation; ${ }^{5}$ Moscow Institute of Physics and Technology, Dolgoprudny, Moscow Region, Russian Federation; ${ }^{6}$ Atlas Oncology Diagnostics, Moscow, Russian Federation; ${ }^{7}$ Center for Personalized Medicine, Children's Hospital Los Angeles and Spatial Sciences Institute, University of Southern California, Los Angeles, California, USA and ${ }^{8}$ AA Kharkevich Institute for Information Transmission Problems RAS, Moscow, Russian Federation Correspondence: Dr S Bruskin, Laboratory of Functional Genomics, Vavilov Institute of General Genetics, Gubkina Street 3, Moscow 119991, Russian Federation.
}

E-mail: sergey.bruskin@gmail.com

Received 10 March 2016; revised 6 May 2016; accepted 24 May 2016 
the development of the disease. To identify transcriptional regulators of psoriatic pathology, we utilized two knowledgebased tools, MetaCore ${ }^{2}$ and cisExpress. ${ }^{3,4}$ Modulation of the identified signaling pathways may be a promising approach for the development of novel management strategies of psoriasis and other diseases commonly associated with this condition..$^{5-7}$

\section{MATERIALS AND METHODS}

\section{Ethics statement}

All steps of data collection and analysis were conducted according to the Declaration of Helsinki principles. Informed written consent was obtained from human subjects under protocols approved by the Ethics Committee of the Vavilov Institute of General Genetics, Russian Academy of Sciences.

\section{Patients and samples}

The patients in this study were unrelated Caucasian individuals with the plaque form of psoriasis from the Bryansk regional STD and Dermatology Center. The metadata for the patients are presented in Supplementary Table S1. Two 4-mm punch biopsy specimens were taken from the skin of the patients, one from the lesional area of the skin (LS sample) and another from the non-lesional area of skin $3-4 \mathrm{~cm}$ apart from the lesion, in an area that did not have any visual signs of psoriasis (NL sample). Patients did not obtain any systemic or PUVA/UV treatment for 1 month before the biopsies. All biopsy samples were immediately transferred to the liquid nitrogen until RNA extraction.

\section{RNA sequencing}

A TissueLyser LT homogenizer (Qiagen, Valencia, CA, USA) was used to homogenize the biopsy specimens. Total RNA was extracted with the ExtractRNA reagent (Evrogen, Moscow, Russia) according to the manufacturer's protocol. Isolated RNA was dissolved in RNase-free water, rRNA was depleted using a RiboMinus Eukaryote Kit for RNA-seq (Life Technologies, Thermo Fisher Scientific, Waltham, MA, USA) and the samples were stored at $-80^{\circ} \mathrm{C}$. The quality of total RNA was evaluated with an RNA 6000 Pico Chip Kit and an Agilent 2100 Bioanalyzer (Agilent Technologies Inc., Santa Clara, CA, USA) and with a Quant-iT RNA Assay Kit and a Qubit fluorometer (Life Technologies). The average RNA integrity number of the samples was $\geqslant 7$. Library preparation and sequencing were performed using a SOLiD 4 System platform and sequencing chemistry according to the manufacturer's instructions (Life Technologies).

\section{Processing and mapping of RNA-seq reads and differential expression analysis}

Raw pair-end reads $(50+25 \mathrm{bp})$ were obtained from the SOLID4 System (Applied Biosystems) in color space format (*.csfasta) and were filtered for quality. The adaptor sequences were trimmed, and the reads were aligned to the UCSC human reference genome (hg19) using the Applied Biosystems Bioscope software (Applied Biosystems Bioscope, Thermo Fisher Scientific, Waltham, MA, USA) to obtain reads in the BAM format. Mapping to multiple locations was permitted. The aligned read BAM files were assembled into transcripts, their abundance was estimated and tests for differential expression were processed using the Bioconductor DESeq package (Simon Anders, EMBL, Heidelberg, Germany). ${ }^{8}$ False discovery rate
(FDR) correction for multiple testing was performed according to Benjamini et al. ${ }^{9-11}$

\section{Pathway analysis and identification of transcriptional regulators}

Using a fold change cutoff (FC) $>1.5$ and a FDR $<0.05$ and including only those genes that had reads at all samples, we identified 1564 DEGs: 938 of them were upregulated and 626 were downregulated. This subset of DEGs was used for gene ontology (GO) analysis using the DAVID tool (Database for Annotation, Visualization and Integrated Discovery ver. 6.7 $)^{12}$ and pathway analysis as well as interactome analysis using the MetaCore database from Thomson Reuters (ver. 6.11, build 41105, GeneGo, Thomson Reuters, New York, NY, USA). ${ }^{2}$ The MetaCore Pathway analysis tool was used to perform gene network enrichment analysis; the MetaCore Interactome tool was used for the identification of transcriptional regulators of DEG-enriched pathways. ${ }^{2}$ The transcriptional regulators of DEGs were ranked by $Z$-score (representing the level of connectivity) and calculated as follows:

$$
Z-\text { score }=\frac{r-n \frac{R}{N}}{\sqrt{n\left(\frac{R}{N}\right)\left(1-\frac{R}{N}\right)\left(1-\frac{n-1}{N-1}\right)}}=\frac{r-\mu}{\sigma}, \text { where }
$$

$N$ is the total number of nodes (genes) after filtration; $R$ is equal to either the number of nodes in the input list or the nodes associated with the experimental data; $n$ is the number of the nodes in the network; $r$ is the number of the network's nodes associated with the experimental data or included in the input list; $\mu$ and $\sigma$ are the mean and dispersion of the corresponding hypergeometric distribution, respectively.

The cisExpress algorithm ${ }^{3,4}$ was used for the identification of promoter motifs and the discovery of cis-elements in promoter sequences that are statistically associated with the expression patterns of DEG. Promoter sequences were obtained from the EPDnew database, ${ }^{13}$ which is a collection of experimentally validated promoters in the human, mouse, fruit fly and zebrafish genomes. Evidence comes from transcription start site mapping from high-throughput experimental techniques, such as $\mathrm{CAGE}^{14}$ and Oligocapping. ${ }^{15}$ The positions of the promoters were validated using the NPEST algorithm. ${ }^{16}$ We identified 16542 Homo sapiens promoters with corresponding RNA-seq gene expression measurements in lesional and non-lesional skin.

The relative expression (R.E.) values for every gene were calculated from average gene expression data for lesional $(n=14)$ and non-lesional $(n=14)$ skin according to the formula:

$$
\text { R.E. }=\ln \left(\frac{\langle\text { non }- \text { lesional expression }\rangle}{\langle\text { lesional expression }\rangle}\right) .
$$

The $Z$-score for cisExpress data were calculated as follows:

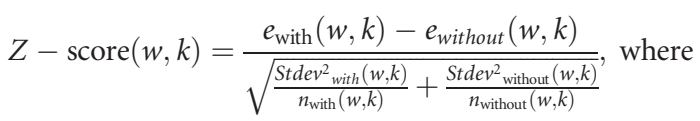

$e_{\text {with }}(w, k)$ and $e_{\text {without }}(w, k)$ are average gene expression values; $\operatorname{Stdev}_{\text {with }}(w, k)$ and $\operatorname{Stdev}_{\text {without }}(w, k)$ are s.d. of gene expression values; and $n_{\text {with }}(w, k)$ and $n_{\text {without }}(w, k)$ are the number of sequences of genes containing and not containing the motif $w$ in the $k$ th window.

\section{FOXA1 target gene identification in the DEG list}

The FOXA1 target list was obtained by merging ChIP-seq data from GSE39241 $1^{17}$ and GSM1099031 ${ }^{18}$ using the edgeR ${ }^{19}$ and DESeq ${ }^{8}$ 
packages with default parameters and then cross-referencing the results with the DEG list.

\section{RESULTS}

\section{Analysis of psoriasis-associated DEGs}

In this study, we identified 1564 genes that were differentially expressed in psoriatic lesions (psoriatic DEGs): 938 of them were upregulated and 626 were downregulated. Analysis of the top 20 upregulated DEGs (Supplementary Table S2) highlighted the importance of immune defense mechanisms, inflammatory response, taxis and chemotaxis of immune cells and alterations of epidermal differentiation in the pathogenesis of psoriasis. Our analysis suggests that the genes with the largest magnitude of expression changes are the 'response' genes that contribute to the pathophysiological manifestations of psoriasis rather than the initiation of the disease. A majority of the top 20 downregulated genes (Supplementary Table S3) were linked to lipid biosynthesis and lipid metabolism. Interestingly, among the top 20 downregulated genes, we detected a number of poorly characterized expression units, including possible pseudogenes, and non-coding RNAs. It remains an open question whether non-coding RNA enrichment is of any functional significance or just an indication of technology bias.

To identify the molecular basis of psoriatic pathology, GO analysis and MetaCore-guided pathway analysis were performed. ${ }^{2}$ The results of the GO analysis (Supplementary

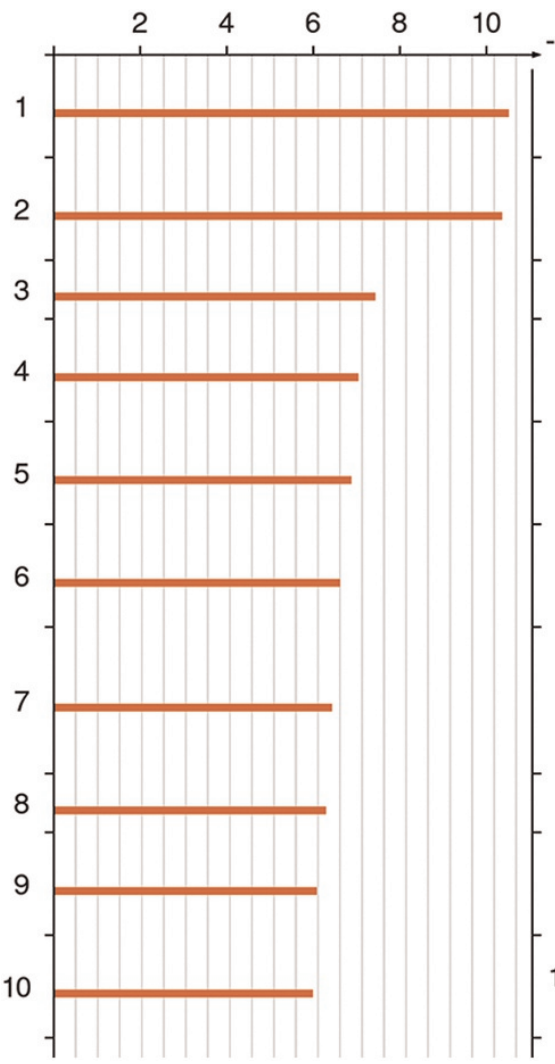

Table S4) generally supported the findings obtained with MetaCore pathway enrichment (Figure 1) and the analysis of top DEGs described above. A detailed discussion of a number of psoriasis-associated pathways is presented in the 'Discussion' and 'Supplementary Materials' sections.

\section{Transcriptional regulation}

The skin serves as a first line of defense against pathogen invasion. The stimulation of different pathogen-sensing receptors (such as pathogen-recognition receptors) leads to the activation of antimicrobial defense (i.e. defensins and other gene clusters, Supplementary Figures S1 and S2, Supplementary Table S5) that is orchestrated by a number of key transcription factors (TFs), including nuclear factor kappa $\mathrm{B}(\mathrm{NF \kappa B})$, activator protein $1, \mathrm{cAMP}$ response element-binding protein, interferon-regulatory factors (IRF) and others. Although the putative antigen leading to the activation of pro-inflammatory signaling in psoriasis has not yet been identified, it is widely accepted that the signaling cascades activated in the course of psoriatic inflammation are mainly the same as those stimulated during pathogen invasion. The activation of inflammatory and antiapoptotic proteins ultimately alerts the immune system of the invasion and induces the recruitment of leukocytes to the site of infection. $^{20}$

To ascertain the key regulatory 'hub points' of the psoriatic networks, two independent approaches were used. The first of

$-\log (p$ Value $)$

1. Immune response_T cell subsets: cell

surface markers

2. Immune response_Inhibitory PD-1 signaling in T cells

3. Th17 cells in CF

4. Immune response_IFN alpha/beta signaling pathway

5. Immune response_IL-13 signaling via JAK-STAT

6. Immune response_Differentiation and clonal expansion of CD8+ T cells

7. Immune response_T regulatory cellmediated modulation of antigenpresenting cell functions

8. Th17 cells in CF (mouse model)

9. Immune response_Immunological synapse formation

10. Immune response_IL-17 signaling pathways

Figure 1 Top 10 DEG-enriched signaling pathways. Sorted by statistical significance of the findings. The results were obtained using the MetaCore pathway analysis tool (GeneGO/Thomson Reuters). 
them utilizes the MetaCore Interactome enrichment tool ${ }^{2}$ that evaluates the levels of connectivity between the nodes (that can be either proteins or genes), identifies overconnected nodes and, according to the node function, suggests possible transcriptional regulators that drive the observed pattern of gene expression in the entire data set. The second approach relies on the cisExpress algorithm, ${ }^{3,4}$ which allows one to perform de novo discovery of the motif within the putative promoter regions of DEGs by means of comparison of these regions with the content of the HOCOMOCO ${ }^{21} \mathrm{v} 9$, JASPAR, ${ }^{22}$ HumanTF $1.0^{23}$ and footprint $\mathrm{DB}^{24}$ databases of known TF-binding sites (TFBSs). Next the identified lists of transcriptional regulators were compared with the data compiled by Swindell et al. ${ }^{25}$ using meta-analysis of transcriptomes of 237 psoriatic patients, a dictionary of 2935 putative TFBSs and the sites for unconventional DNA-binding proteins. Swindell et $a .^{25}$ identified psoriasis-response elements that were overrepresented upstream of psoriasis DEGs in putative promoters that were defined as sequences starting at $5 \mathrm{~kb}$ upstream and ending at $500 \mathrm{bp}$ downstream from the major transcription start site.

MetaCore-guided identification of transcriptional regulators. The MetaCore Interactome tool determines the density of interactions between each protein from a data set of interest

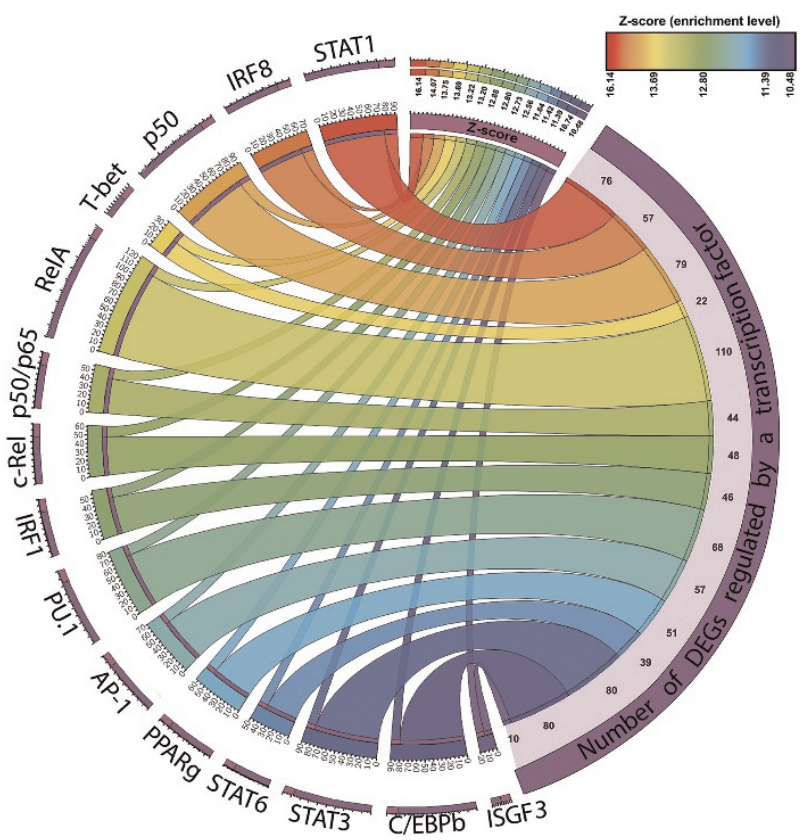

Figure 2 Top 15 transcriptional regulators of DEG genes (identified with MetaCore Interactome). Transcription factors are ranked according to their Z-score (the level of connectivity of the TF to the DEG list). Larger Z-scores represent higher levels of connectivity between the transcription factor and the DEG list. The colors from red to violet indicate $Z$-score values from higher (16.14) to lower (10.48). The width of the ribbon for the transcription factor corresponds to the number of target genes for the transcription factor in the DEG list (for example, for the STAT1 gene, the Z-score is 16.14 , and the number of regulated DEGs is 76). The numerical data for this figure are in Supplementary Table S6. and all other proteins, evaluates statistically significant interactions within the set and analyzes the functions of the selected interacting proteins. ${ }^{2}$ Because proteins usually work in groups (such as protein complexes and pathways) that are defined by protein interactions, it is assumed that the relative connectivity of each hub reflects its relevance, or importance, and it may be used for the identification of transcriptional regulators of DEG-enriched signaling cascades. Even if the expression levels of the mRNA encoding the TF itself are not altered, for example, when the TF in question is predominantly regulated posttranscriptionally, the number of targets it interacts with depends on the state of its activation or suppression. Hence, the enrichment or the depletion of an interacting protein network indicates the activation or suppression of the TF that orchestrates the network.

We identified possible transcriptional regulators of DEGs (the TFs significantly associated with DEG list; Supplementary Table S6) and computed their $Z$-score-the degree of connectivity of the TF to the list of DEGs. The $Z$-score signifies a number of connections obtained between a TF and a list of DEGs in excess of the number of connections expected with a random set of genes of the same size. The identified 'Top' transcriptional regulators of the DEGs (Figure 2) are ranked according to their $Z$-scores corresponding to the influence of the TFs on the pathological processes associated with the disease. Their regulation leads to the development of the main distinctive features of psoriasis. In the 'Top' TF list, there are both cell type-specific TFs (for example, PU.1, which is a master regulator of myeloid cells ${ }^{26}$ ) and ubiquitously expressed TFs associated with inflammatory pathways (for example, $\mathrm{NF \kappa B}$ and $\mathrm{IRF}^{27}$ ), which reflects alterations in cell populations in a plaque compared with the unaffected skin. In addition to the TFs commonly associated with psoriasis, we identified TFs that have not been previously associated with the disease (Supplementary Table S7).

Further analysis is needed to determine the roles of the identified DEGs in disease progression. To illustrate our hypothesis of their involvement in psoriasis, we analyzed one of the TFs, FOXA1, and its target genes, looking for possible associations with pathogenesis. Using the CHIP-seq data available from experiments GSE39241 ${ }^{17}$ and GSM1099031, ${ }^{18}$ we have compiled a list of gene targets for this TF and compared it with the list of DEGs identified by RNA-seq (Supplementary Table S12). The intersection between the two lists was used in the GO enrichment analysis to reveal processes in psoriatic lesions regulated by this TF (Supplementary Table S13). FOXA1 was found to be associated with the immune alterations indicative for the disease as well as with the abnormalities of lipid metabolism.

De novo analysis of transcriptional regulation of DEGs using the cisExpress algorithm. Another approach that we utilized for the identification of the transcriptional regulators of DEGs was based on the cisExpress algorithm. ${ }^{3,4}$ cisExpress finds putative regulatory elements using a combination of sequence and expression information. We used 16542 validated $H$. sapiens 
promoters that have corresponding RNA-seq gene expression measurements in lesional and non-lesional skin.

The length of a promoter region varies from gene to gene, and the identification of a 'promoter window' containing the most important regulatory sequences for each gene is a separate challenge. Hence, for our analysis, we used the 'core promoter$5^{\prime}$ untranslated region' of $(+500,-500)$ base pairs around the transcription start site of each gene. The analyzed set of the promoters was examined for the presence of motifs (putative TFBSs), and the corresponding gene expression values were compared for genes whose promoters did and did not contain the motifs using a $t$-test. We compiled a ranked list of $>100$ position-specific motifs in the promoter regions of DEGs (Supplementary Table S8).

The top 10 motifs in the ranked list have the highest influence on gene expression (Table 1). For every motif, a $Z$-score was calculated. Positive $Z$-score values suggest that the presence of the motif is associated with elevated levels of gene expression in non-lesional skin, while negative values show that the presence of the motif is associated with elevated levels of gene expression in lesional skin. The absolute value of the $Z$-score is used to calculate the confidence level of the influence of the motif on gene expression. The complete list of motifs is given in Supplementary Table S9.

The motifs were examined for similarity with known TFBSs. HOMOCO v9, ${ }^{21}$ JASPAR 2014, ${ }^{22}$ HumanTF $1.0^{23}$ and footprintDB ${ }^{24}$ databases were used for this analysis (see Supplementary Table S9). For example, one of the identified motifs (AAGATG) is related to the ETS1 TF, a negative regulator of Th17 cells, ${ }^{28}$ which are one of the most important T-cell populations in psoriasis, and to GATA-3, a transcriptional regulator leading to $\mathrm{T}$ helper type 2 (Th2) polarization in the Th1/Th2-cell-type switch, ${ }^{29}$ which is downregulated in psoriatic skin. The CCGGAA motif is associated with the TF ELK4, which is highly expressed in lesional psoriatic skin. ${ }^{30}$

Identification of the key transcriptional regulators of the psoriatic transcriptome. To find the key transcriptional regulators of the DEGs, we compared lists identified by the two computational approaches (MetaCore and cisExpress) and the results of Swindell et al. ${ }^{25}$ Comparison of the three groups of transcriptional regulators (327 cisExpress-identified, 200 MetaCoreidentified and 439 identified by Swindell et al.) (Figure 3) identified 42 common TFs representing the 'core' TF regulators of the psoriatic transcriptome (Table 2).

The majority of elements of the 'core TF' list are TFs associated with inflammation (NFKB, IRF9, JUN, FOS, SRF), the activity of T-cells in the psoriatic lesions (STAT6, FOXP3, NFATC2, GATA3, TCF7, RUNX1 etc.), hyperproliferation and migration of keratinocytes (JUN, FOS, NFIB, TFAP2A, TFAP2C) and lipid metabolism (TFAP2, RARA, VDR). There were several FOX ('fork-head' box) family proteins in the list containing the evolutionary conserved 'fork-head' or 'winged-helix' DNA-binding domain. These proteins could work as active regulators of cell proliferation and metabolism and also serve as pioneer factors that de-condense chromatin, therefore facilitating the binding of other sequence-specific TFs to target enhancers, repressors and promoters, wiring global gene networks essential for cell fate decisions. ${ }^{31}$ We also found 294 DEG-associated TFs not identified by Swindell et al. ${ }^{25}$ Sixty-six of them were identified by cisExpress as well as by MetaCore (Figure 3 and Supplementary Table S10). Among the negative regulators, there is the CAGAC motif, frequently found in Smadresponsive promoter regions. Smad TFs have a key role in cytokine signaling pathways. The Smad complex includes DNA-binding cofactors influencing the recruitment of transcriptional coactivators or corepressors. ${ }^{32}$ Another TFBS with a negative association with gene expression in psoriatic lesions is the CACCC-box, a biding site for the Krüppel-like factors that can act as a transcriptional repressor. ${ }^{33-37}$ Recently, Krüppel-like factors were reported to regulate epidermal proliferation and differentiation in humans and control the physiological reaction to counteract the abnormal differentiation and proliferation of keratinocytes. ${ }^{38}$

\section{Pathway analysis}

To investigate the relationships between the DEGs, we performed gene network enrichment analysis using the MetaCore software. The top 10 signaling networks enriched with DEGs were mainly associated with different alterations in immune signaling present in the psoriatic lesions (Figure 1), for example, the map 'Immune response_IL-17 signaling pathway' (Figure 4). This agrees with the hypothesis that the main feature of psoriasis is the cytokine storm and altered balance of cytokines, chemokines and growth factors regulating various immune and inflammatory responses (see Supplementary Materials for a detailed discussion). The main psoriasisassociated transcriptional regulators of this map are $\mathrm{NF \kappa B}$ and $\mathrm{C} / \mathrm{EBPb}$, as defined by the overexpression of their target genes shown on the map (for example, CCL2, CCL7, CCL20, HBD2, IL8, iNOS2 and so on) and the consequent activation of downstream processes important for disease pathogenesis (chemotaxis of neutrophils, dendritic and $\mathrm{T}$ cells, alterations in cell adhesion and antibacterial activity). All of the TFs on the map were identified in the Top15 list in MetaCore analysis (Figure 2) as well as in the cisExpress analysis (Supplementary Table S8).

\section{DISCUSSION}

In psoriasis, the observed changes in the gene expression levels and the DEG enrichment of certain GO processes may be explained by two different disease-associated phenomena: changes in the transcription and degradation rates of mRNA and alterations in the composition of cells within the lesion, which is usually characterized by epidermal thickening, the accumulation of immune cells and thinning of the subcutaneous fat layer. Therefore, whether the differential expression observed between lesional and non-lesional psoriatic samples truly reflects alterations in intracellular signaling remains unclear. ${ }^{37}$ The top 10 signaling pathways (Figure 1) highlighted the importance of the activation and chemotaxis of 
Table 1 Top 10 motifs with the highest confidence of influence on gene expression identified with cisExpress

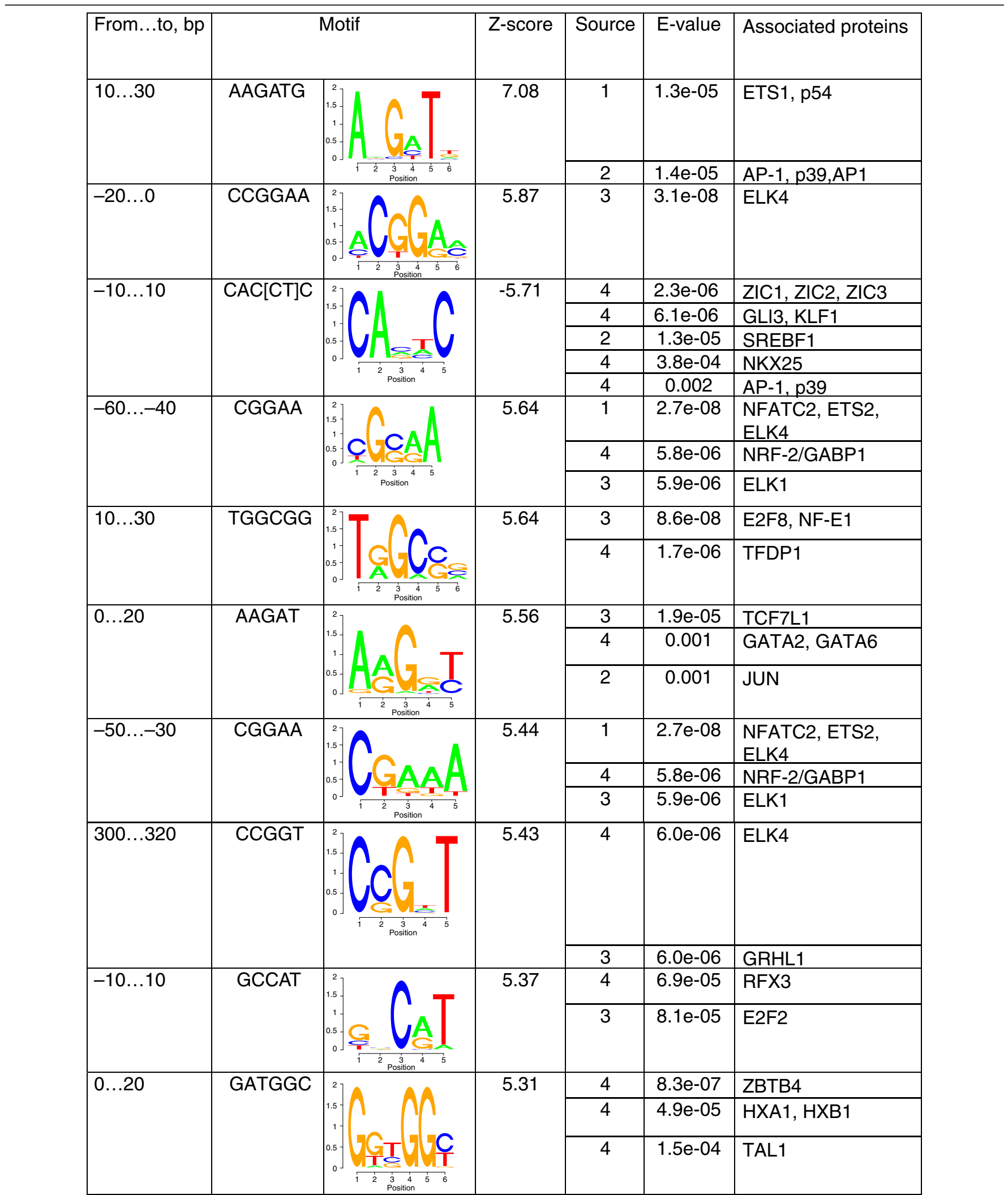

'From . to' is the position of the 'window' where the motif was discovered relative to the gene transcription start. Source: 1 , footprintDB.20 2, JASPAR.18 3, HumanTF $1.0 ;{ }^{19} 4$, HOCOMOCO; ${ }^{17}$ Positive $Z$-score values suggest that the presence of the motif promotes the expression of a gene in non-lesional skin, while negative $Z$-score values suggest that the motif acts in lesional skin. 


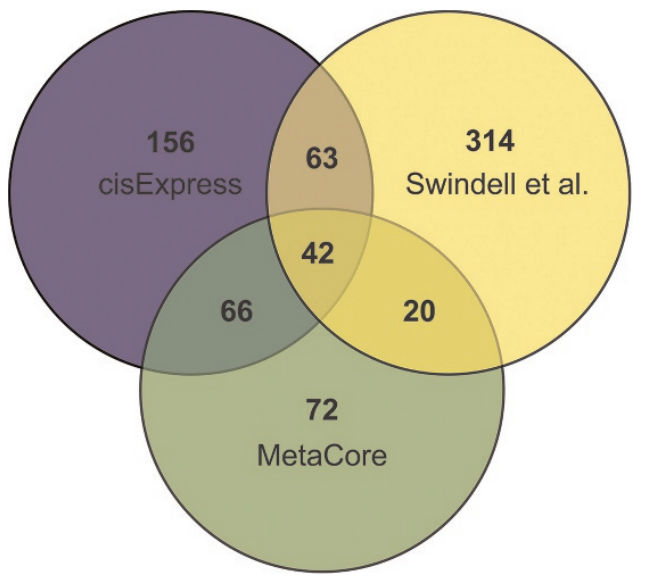

Figure 3 Venn diagram showing overlap between lists of transcriptional regulators of DEG. green, identified by the MetaCore Interactome software; Violet, TFs identified by the cisExpress tool; yellow, identified in Swindell et al. ${ }^{25}$

immune cells mediated by the locally enhanced production of pro-inflammatory cytokines and chemokines and increasing inflammation. The importance of different populations of $\mathrm{T}$ cells in the pathogenesis of the disease is illustrated by the DEG-enriched map 'Immune response: T-cell subsets secreted signals' (Supplementary Figure S3). It shows the shift of T-cell populations toward interleukin-17-producing types, indicating the activation and enhanced migration of psoriasis-specific populations of $\mathrm{T}$ cells to the lesional skin.

One of the main regulators of $\mathrm{T}$-cell polarization and population maintenance is the regulatory T-cell (Treg) population (Supplementary Figure S4). Alterations in the presence and activity of Tregs in the skin of psoriasis patients may trigger development of the disease. ${ }^{39}$ The canonical transcriptional regulator of this cell population is the TF FOXP3. A recent study described a new population of Treg cells that carry a non-canonical marker, FOXA1, instead of the canonical FOXP3. ${ }^{40}$ This population also has a suppressive role in autoimmunity as adoptive transfer of such cells inhibited experimental autoimmune encephalomyelitis in a FOXA1-and PD-L1-dependent manner. ${ }^{40}$ Our analysis identified the upregulation of FOXP3 $(\mathrm{FC}=2.30, \mathrm{FDR}=0.001)$ and downregulation of FOXA1 $(\mathrm{FC}=0.49, \mathrm{FDR}=0.01)$ in psoriatic lesional skin. We found that most of the markers of Treg cells were overexpressed in the analyzed transcriptomic data $(C D 4$, CD47, CD69, PD-L1) except for FOXA1 itself. We hypothesize that the observed reduced expression of FOXA1 represents differences between Treg populations in non-lesional and lesional psoriatic skin. We suggest that the inhibition of maturation of naive $\mathrm{T}$ cells into a FOXA1+ Treg subpopulation $(C D 4+F O X A 1+C D 47+C D 69+P D-L 1(h i) F O X P 3-)$ contributes to the development of the disease.

The TF FOXA1 is not T-cell specific; it is involved in the regulation of cell differentiation under normal and pathological conditions (in epithelial, prostate and breast cancers; in epithelial cells of the intestines and lungs; and in pancreatic cells and other tissues ${ }^{41-47}$ ). During embryonic stem cell development and hepatic differentiation, FOXA1 functions as a 'pioneer TF' owing to its ability to engage condensed chromatin, bind nucleosome-assembled FOXA1 regulatory elements and displace repressive linker histones in response to retinoic acid treatment and transforming growth factor- $\beta$ signaling. ${ }^{48}$

In addition to its role in hepatic epithelial differentiation, FOXA1 is also involved in uroepithelial differentiation. Differentiation of the urothelium is a process governed by peroxisome proliferator-activated receptor- $\gamma(\operatorname{PPAR}-\gamma)$ that involves intermediary TFs such as IRF-1 and FOXA1. siRNA knockdown of either FOXA1 or IRF-1 abrogates the PPAR- $\gamma$-induced induction of late/terminal urothelial differentiation-associated genes. ${ }^{49}$ PPAR- $\gamma$ is also an important regulator of keratinocyte differentiation as well as the formation of the horny layer of the epidermis, which is rich in lipids and important for the barrier function. Treatment of cultured human keratinocytes with ciglitazone, a PPAR- $\gamma$ activator, increases the mRNA levels of involucrin and transglutaminase- 1 . Moreover, topical treatment of hairless mice with ciglitazone or troglitazone increases loricrin, involucrin and filaggrin expression. As $P P A R \gamma$ itself is downregulated according to the RNA-seq data $(\mathrm{FC}=0.39, \mathrm{FDR}=$ 0.0039 ), and it is an upstream regulator of FOXA1 signaling, it could be speculated that aberrant fatty acid metabolism as well as the general metabolic disturbances observed in psoriasis could lead to impaired cell differentiation via the inhibition of PPARg and the associated inhibition of FOXA1 expression in the skin.

Another possible consequence of reduced FOXA1 expression is disturbed keratinocyte differentiation (more discussion on this topic is in the Supplementary Materials). To evaluate the putative contribution of FOXA1 regulation to the development of the psoriatic process, we have compared lists of FOXA1 targets, identified in ChIP-seq experiments by Hurtado et al., ${ }^{50}$ with the DEGs identified by our RNA-seq analysis. We demonstrated that FOXA1 is a transcriptional regulator of the top DEGs that serve as major histopathological contributors (Supplementary Table S2), encoding S100 proteins, serpins and genes for chemoattractant CXCL proteins. Among other important upregulated targets contributing to the disease were HLA-DPB1 (an HLA class II beta chain paralog expressed in antigen-presenting cells; a risk allele for the disease ${ }^{51}$ ), keratins 6B and 6C (activation markers of keratinocytes essential for the formation of keratin intermediate filaments that also take part in wound healing) and PPAR (a TF overexpressed in psoriasis that enhances the proliferation of keratinocytes and is induced by JUNB in keratinocytes). ${ }^{52}$ Among the downregulated targets were genes associated with lipid disturbances observed in psoriatic lesions, including known components of fatty acid metabolism such as the acyl-CoA wax alcohol acyltransferase gene $A W A T 2$, the fatty acid elongase gene ELOVL3, the fatty acid-binding protein FABP4 and many others. Ontology analysis of the FOXA1 target DEGs yielded a list of ontologies similar to the whole DEG list discussed above (Supplementary 
Table 2 Core TF regulators of the psoriatic transcriptome

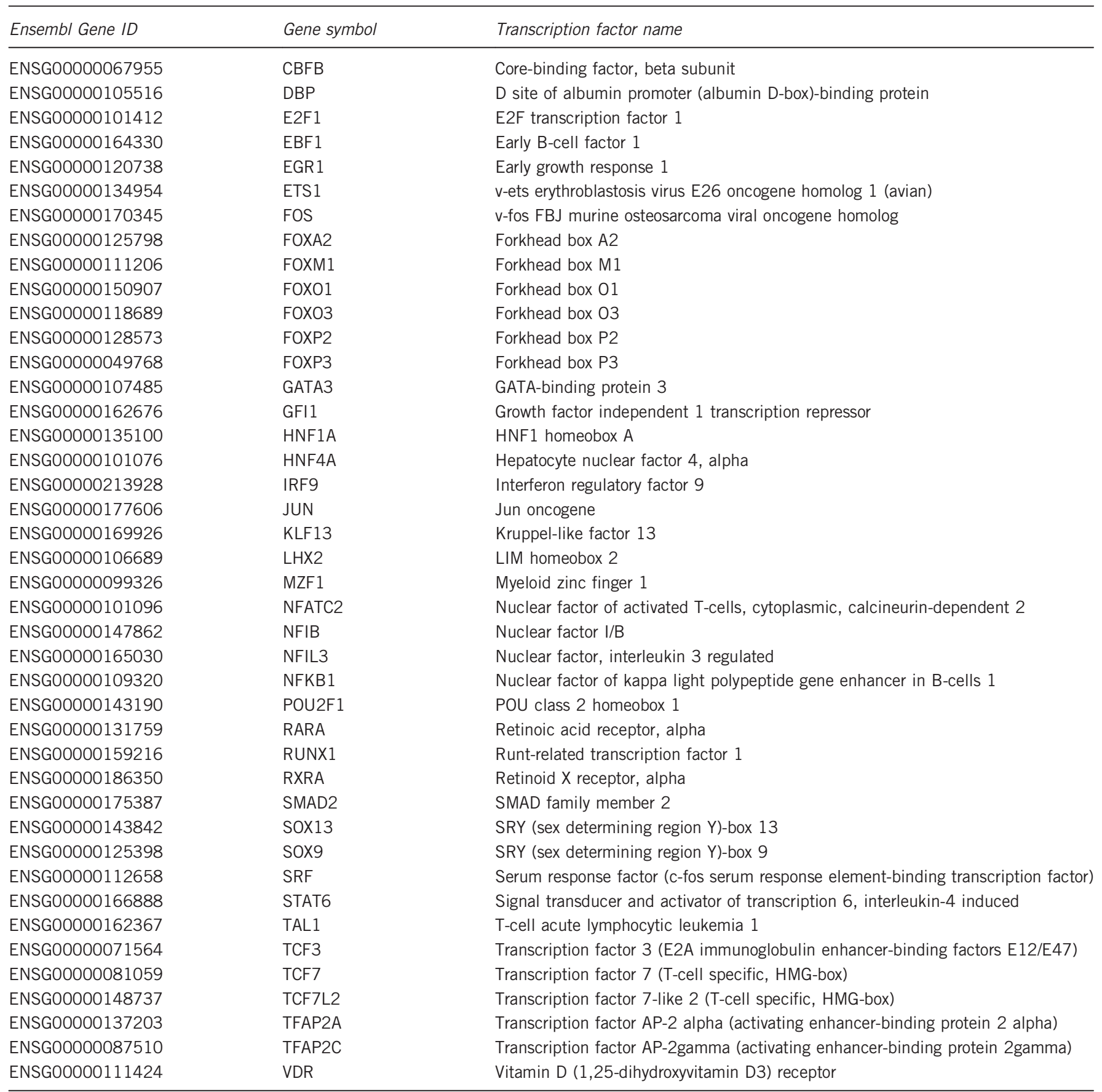

Abbreviation: TF, transcription factor.

Tables S11 and S12). Comparison of the lists of ontologies showed that FOXA1 is a part of a regulatory complex accounting for the most important psoriatic alterations and signaling cascades important for pathology. Thus this TF is a promising candidate for future investigations of psoriasis.

There are two potential limitations of our study: relatively small samples size (14 pairs) and the ethnic homogeneity of samples (only Caucasians). Earlier studies demonstrated that a sample set of this size is sufficient to identify DEGs. ${ }^{53,54}$ Because the RNA sequencing coverage depth corresponds to a log-normal distribution, a parametric test such as $t$-test could be utilized to identify DEGs. ${ }^{55}$ To evaluate the reproducibility of the results, we compared our list of DEGs with a recent large-scale transcriptomic analysis of psoriatic samples (92 samples of lesional skin and 82 samples of non-lesional skin $)^{37}$ that identified a larger number of DEGs (6254 DEGS, 4581 upregulated and 1673 downregulated genes, FC $>1.5$, FDR $<0.05$ ). We demonstrated that $>60 \%$ of the DEGs identified in our study matched the DEGs found by Li et al. ${ }^{37}$ (Supplementary Figure S6). Given the differences 


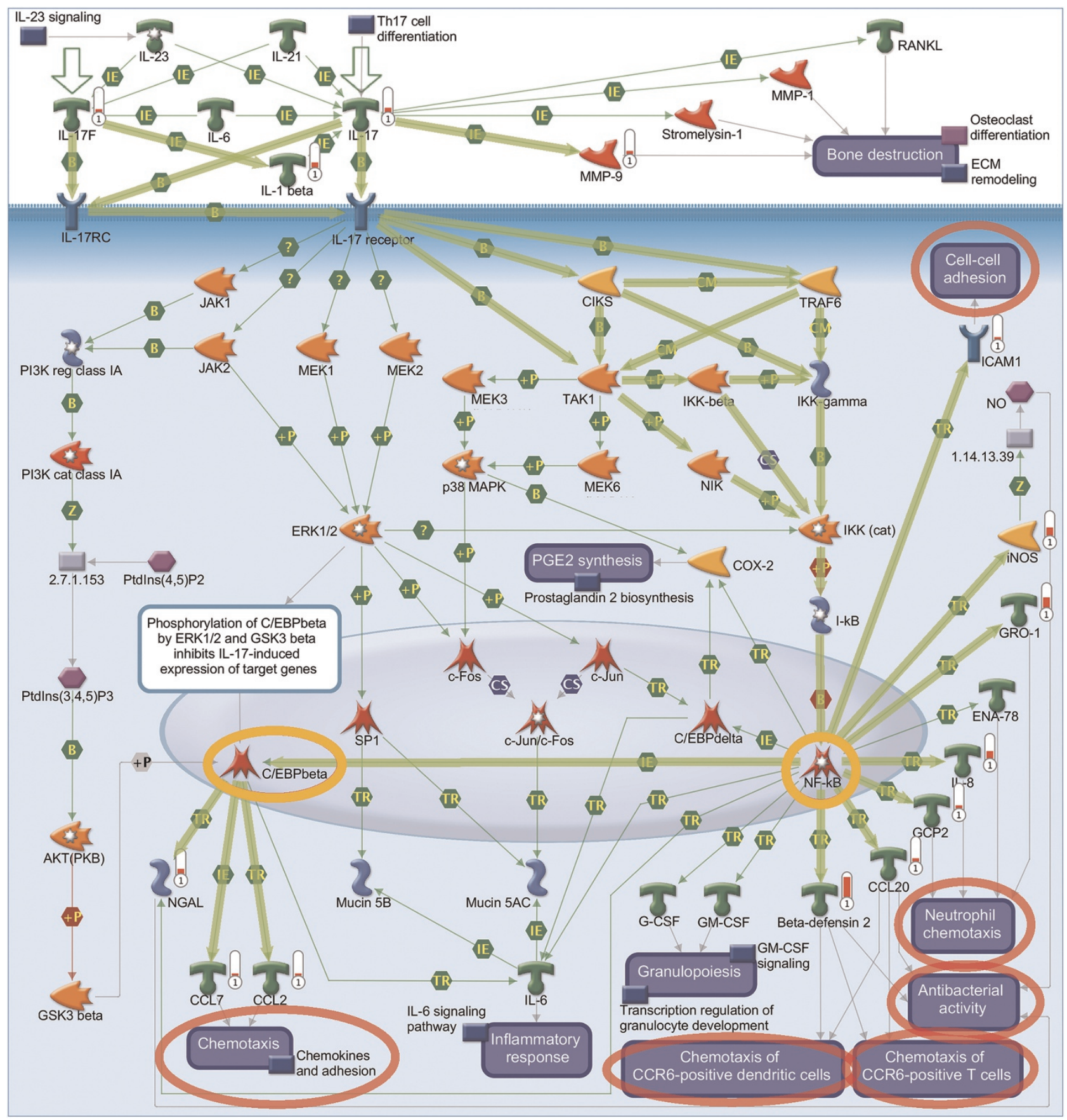

Figure 4 Immune response_IL-17 signaling pathway. Illustration generated with the MetaCore pathway analysis tool (GeneGO/Thomson Reuters) and enriched with DEGs. A description of the map symbols is in Supplementary Figure S5. The red bars near the gene name illustrate the level of gene overexpression. Green arrows indicate signaling cascades enriched with DEGs. Orange circles indicate important transcription regulators of DEGs present in the map. Red circles indicate downstream signaling cascades activated by the pathway in lesional psoriatic skin.

between the sequencing platforms, sample sizes and studied populations, the concordance of $>60 \%$ is truly remarkable.

To avoid admixture, our samples were obtained from Caucasian patients in the regions where the Caucasian population is predominant. A recent large trans-ethnic genome-wide meta-analysis (15369 psoriasis cases and 19517 controls of Caucasian and Chinese ancestries) revealed population-specific effects or allelic heterogeneity for 11 susceptibility loci and noted that such population-specific effects contribute significantly to the ethnic diversity of psoriasis prevalence and may influence the course of disease. ${ }^{56}$ Therefore, our data containing only Caucasian samples allows us to zoom in on immuno-pathogenesis and important psoriasis-associated pathways in Caucasian population. Additional studies should 
be conducted to evaluate the applicability of such data to the different ethnic groups.

In conclusion, our analysis highlighted the importance of immune system alterations in the development of psoriasis. We presented a list of identified core transcriptional regulators of the psoriatic transcriptome that can be validated to provide insight into the mechanisms of gene regulation in psoriasis. We also identified novel transcriptional regulators of psoriasisassociated pathways previously not implicated in the pathology. The comparison of our data with public ChIP-seq data allowed us to suggest a hypothesis explaining the role of the TF FOXA1 in psoriasis.

\section{CONFLICT OF INTEREST}

The authors declare no conflict of interest.

\section{ACKNOWLEDGEMENTS}

The reported study was funded by The Russian Foundation for Basic Research (AZ was supported by 16-34-01356 mol_a and AM was supported by 14-04-01207A). TVT was supported by grants from The National Institute for General Medical Studies (GM068968), the Eunice Kennedy Shriver National Institute of Child Health and Human Development (HD070996) and the National Science Foundation Division of Evolutionary Biology (1456634).

1 Quigley D. RNA-seq permits a closer look at normal skin and psoriasis gene networks. J Invest Dermatol 2014; 134: 1789-1791.

2 Bessarabova M, Ishkin A, JeBailey L, Nikolskaya T, Nikolsky Y. Knowledge-based analysis of proteomics data. BMC Bioinformatics 2012; 13(Suppl 16): S13.

3 Triska M, Grocutt D, Southern J, Murphy DJ, Tatarinova T. cisExpress: motif detection in DNA sequences. Bioinformatics 2013; 29: 2203-2205.

4 Troukhan M, Tatarinova T, Bouck J, Flavell RB, Alexandrov NN. Genome-wide discovery of cis-elements in promoter sequences using gene expression. OMICS 2009; 13: 139-151.

5 Mayer TZ, Simard FA, Cloutier A, Vardhan H, Dubois CM, McDonald PP. The p38-MSK1 signaling cascade influences cytokine production through CREB and C/EBP factors in human neutrophils. J Immunol 2013; 191: 4299-4307.

6 Liu B, Sun L, Liu Q, Gong C, Yao Y, Lv X et al. A cytoplasmic NF-kappaB interacting long noncoding RNA blocks IkappaB phosphorylation and suppresses breast cancer metastasis. Cancer Cell 2015; 27: 370-381.

7 Pallandre JR, Brillard E, Crehange G, Radlovic A, Remy-Martin JP, Saas P et al. Role of STAT3 in CD4+CD25+FOXP3+ regulatory lymphocyte generation: implications in graft-versus-host disease and antitumor immunity. J Immunol 2007; 179: 7593-7604.

8 Anders S, Huber W. Differential expression analysis for sequence count data. Genome Biol 2010; 11: R106.

9 Benjamini Y, Heller R. Screening for partial conjunction hypotheses. Biometrics 2008; 64: 1215-1222.

10 Benjamini Y, Drai D, Elmer G, Kafkafi N, Golani I. Controlling the false discovery rate in behavior genetics research. Behav Brain Res 2001; 125: 279-284.

11 Benjamini Y, Hochberg Y. Controlling the false discovery rate: a practical and powerful approach to multiple testing. J R Stat Soc B 1995; 57: 289-300.

12 Huang, da W, Sherman BT, Lempicki RA. Systematic and integrative analysis of large gene lists using DAVID bioinformatics resources. Nat Protoc 2009; 4: 44-57.

13 Dreos R, Ambrosini G, Cavin Perier R, Bucher P. EPD and EPDnew, high-quality promoter resources in the next-generation sequencing era. Nucleic Acids Res 2013; 41: D157-D164.
14 Takahashi H, Kato S, Murata M, Carninci P. CAGE (cap analysis of gene expression): a protocol for the detection of promoter and transcriptional networks. Methods Mol Biol 2012; 786: 181-200.

15 Maruyama K, Sugano S. Oligo-capping: a simple method to replace the cap structure of eukaryotic mRNAs with oligoribonucleotides. Gene 1994; 138: 171-174.

16 Tatarinova T, Kryshchenko A, Triska M, Hassan M, Murphy D, Neely M et al. NPEST: a nonparametric method and a database for transcription start site prediction. Quant Biol 2013; 1: 261-271.

17 Caravaca JM, Donahue G, Becker JS, He X, Vinson C, Zaret KS. Bookmarking by specific and nonspecific binding of FoxA1 pioneer factor to mitotic chromosomes. Genes Dev 2013; 27: 251-260.

$18 \mathrm{Ni} \mathrm{M}$, Chen Y, Fei T, Li D, Lim E, Liu XS et al. Amplitude modulation of androgen signaling by c-MYC. Genes Dev 2013; 27: 734-748.

19 Robinson MD, McCarthy DJ, Smyth GK. edgeR: a Bioconductor package for differential expression analysis of digital gene expression data. Bioinformatics 2010; 26: 139-140.

20 Hodgson A, Wan F. Interference with nuclear factor kappaB signaling pathway by pathogen-encoded proteases: global and selective inhibition. Mol Microbiol 2016; 99: 439-452.

21 Kulakovskiy IV, Medvedeva YA, Schaefer U, Kasianov AS, Vorontsov IE, Bajic VB et al. HOCOMOCO: a comprehensive collection of human transcription factor binding sites models. Nucleic Acids Res 2013; 41: D195-D202.

22 Mathelier A, Zhao X, Zhang AW, Parcy F, Worsley-Hunt R, Arenillas DJ et al. JASPAR 2014: an extensively expanded and updated open-access database of transcription factor binding profiles. Nucleic Acids Res 2014; 42(Database issue): D142-D147.

23 Jolma A, Yan J, Whitington T, Toivonen J, Nitta KR, Rastas P et al. DNA-binding specificities of human transcription factors. Cell 2013; 152: 327-339.

24 Kirsanov DD, Zanegina ON, Aksianov EA, Spirin SA, Karyagina AS, Alexeevski AV. NPIDB: Nucleic acid-Protein Interaction DataBase. Nucleic Acids Res 2013; 41: D517-D523.

25 Swindell WR, Sarkar MK, Stuart PE, Voorhees JJ, Elder JT, Johnston A et al. Psoriasis drug development and GWAS interpretation through in silico analysis of transcription factor binding sites. Clin Trans/ Med 2015; 4: 13.

26 Vangala RK, Heiss-Neumann MS, Rangatia JS, Singh SM, Schoch C, Tenen DG et al. The myeloid master regulator transcription factor PU.1 is inactivated by AML1-ETO in t(8;21) myeloid leukemia. Blood 2003; 101: 270-277.

27 Iwanaszko M, Kimmel M. NF-kappaB and IRF pathways: cross-regulation on target genes promoter level. BMC Genomics 2015; 16: 307.

28 Lee PH, Puppi M, Schluns KS, Yu-Lee LY, Dong C, Lacorazza HD. The transcription factor E74-like factor 4 suppresses differentiation of proliferating CD4+ T cells to the Th17 lineage. J Immunol 2014; 192: 178-188.

29 Zhang P, Chen HX, Duan YQ, Wang WZ, Zhang TZ, Li JW et al. Analysis of Th1/Th2 response pattern for erythrodermic psoriasis. J Huazhong Univ Sci Technolog Med Sci 2014; 34: 596-601.

30 Swindell WR, Johnston A, Carbajal S, Han G, Wohn C, Lu J et al. Genome-wide expression profiling of five mouse models identifies similarities and differences with human psoriasis. PLOS ONE 2011; 6: e18266.

31 Lam EW, Brosens JJ, Gomes AR, Koo CY. Forkhead box proteins: tuning forks for transcriptional harmony. Nat Rev Cancer 2013; 13: 482-495.

32 Massague J, Seoane J, Wotton D. Smad transcription factors. Genes Dev 2005; 19: 2783-2810.

33 van Vliet J, Turner J, Crossley M. Human Kruppel-like factor 8: a CACCCbox binding protein that associates with CtBP and represses transcription. Nucleic Acids Res 2000; 28: 1955-1962.

34 Chen ZY, Shie J, Tseng C. Up-regulation of gut-enriched kruppel-like factor by interferon-gamma in human colon carcinoma cells. FEBS Lett 2000; 477: 67-72.

35 Guy LG, Delvoye N, Wall L. Expression of a human beta-globin transgene in mice with the CACC motif and upstream sequences deleted from the promoter still depends on erythroid Kruppel-like factor. J Biol Chem 2000; 275: 3675-3680.

36 Ulgiati D, Subrata LS, Abraham LJ. The role of Sp family members, basic Kruppel-like factor, and $\mathrm{E}$ box factors in the basal and IFN-gamma regulated expression of the human complement C4 promoter. J Immunol 2000; 164: 300-307. 
37 Li B, Tsoi LC, Swindell WR, Gudjonsson JE, Tejasvi T, Johnston A et al. Transcriptome analysis of psoriasis in a large case-control sample: RNA-seq provides insights into disease mechanisms. J Invest Dermatol 2014; 134: 1828-1838.

38 Kim KJ, Park S, Park YH, Ku SH, Cho EB, Park EJ et al. The expression and role of kruppel-like factor 4 in psoriasis. Ann Dermatol 2014; 26 : 675-680.

39 Keijsers RR, van der Velden HM, van Erp PE, de Boer-van Huizen RT, Joosten I, Koenen $\mathrm{HJ}$ et al. Balance of Treg vs. T-helper cells in the transition from symptomless to lesional psoriatic skin. Br J Dermatol 2013; 168: 1294-1302.

40 Liu Y, Carlsson R, Comabella M, Wang J, Kosicki M, Carrion B et al. FoxA1 directs the lineage and immunosuppressive properties of a novel regulatory T cell population in EAE and MS. Nat Med 2014; 20: 272-282.

41 DeGraff DJ, Grabowska MM, Case TC, Yu X, Herrick MK, Hayward WJ et al. FOXA1 deletion in luminal epithelium causes prostatic hyperplasia and alteration of differentiated phenotype. Lab Investig 2014; 94: 726-739.

42 Grabowska MM, Elliott AD, DeGraff DJ, Anderson PD, Anumanthan G, Yamashita $\mathrm{H}$ et al. NFI transcription factors interact with FOXA1 to regulate prostate-specific gene expression. Mol Endocrinol 2014; 28: 949-964.

43 Albergaria A, Paredes J, Sousa B, Milanezi F, Carneiro V, Bastos J et al. Expression of FOXA1 and GATA-3 in breast cancer: the prognostic significance in hormone receptor-negative tumours. Breast Cancer Res 2009; 11: R40.

44 Ye DZ, Kaestner KH. Foxa1 and Foxa2 control the differentiation of goblet and enteroendocrine L- and D-cells in mice. Gastroenterology 2009; 137: 2052-2062.

45 Yoshimi T, Nakamura N, Shimada S, Iguchi K, Hashimoto F, Mochitate K et al. Homeobox B3, FoxA1 and FoxA2 interactions in epithelial lung cell differentiation of the multipotent M3E3/C3 cell line. Eur J Cell Biol 2005; 84: 555-566.

46 Heddad Masson M, Poisson C, Guerardel A, Mamin A, Philippe J, Gosmain Y. Foxa1 and Foxa2 regulate alpha-cell differentiation, glucagon biosynthesis, and secretion. Endocrinology 2014; 155: 3781-3792.

47 DeGraff DJ, Clark PE, Cates JM, Yamashita H, Robinson VL, Yu X et al. Loss of the urothelial differentiation marker FOXA1 is associated with high grade, late stage bladder cancer and increased tumor proliferation. PLOS ONE 2012; 7: e36669.

48 Taube JH, Allton K, Duncan SA, Shen L, Barton MC. Foxa1 functions as a pioneer transcription factor at transposable elements to activate Afp during differentiation of embryonic stem cells. J Biol Chem 2010; 285: $16135-16144$.

49 Varley CL, Bacon EJ, Holder JC, Southgate J. FOXA1 and IRF-1 intermediary transcriptional regulators of PPARgamma-induced urothelial cytodifferentiation. Cell Death Differ 2009; 16: 103-114.

50 Hurtado CW, Furuta GT, Kramer RE. Etiology of esophageal food impactions in children. J Pediatr Gastroenterol Nutr 2011; 52: 43-46.

$51 \mathrm{Kim}$ TG, Lee HJ, Youn JI, Kim TY, Han H. The association of psoriasis with human leukocyte antigens in Korean population and the influence of age of onset and sex. J Invest Dermatol 2000; 114: 309-313.

52 Romanowska M, al Yacoub N, Seidel H, Donandt S, Gerken H, Phillip S et al. PPARdelta enhances keratinocyte proliferation in psoriasis and induces heparin-binding EGF-like growth factor. J Invest Dermatol 2008; 128: $110-124$.

53 Swindell WR, Remmer HA, Sarkar MK, Xing X, Barnes DH, Wolterink L et al. Proteogenomic analysis of psoriasis reveals discordant and concordant changes in mRNA and protein abundance. Genome Med 2015; 7: 86.

54 Li J, Li X, Hou R, Liu R, Zhao X, Dong F et al. Psoriatic T cells reduce epidermal turnover time and affect cell proliferation contributed from differential gene expression. J Dermatol 2015; 42: 874-880.

55 Yoon S, Xuan Z, Makarov V, Ye K, Sebat J. Sensitive and accurate detection of copy number variants using read depth of coverage. Genome Res 2009; 19: $1586-1592$.

56 Yin X, Low HQ, Wang L, Li Y, Ellinghaus E, Han J et al. Genome-wide metaanalysis identifies multiple novel associations and ethnic heterogeneity of psoriasis susceptibility. Nat Commun 2015; 6: 6916.

(1) (2) (2) This work is licensed under a Creative Commons Attribution-NonCommercial-ShareAlike 4.0 International License. The images or other third party material in this article are included in the article's Creative Commons license, unless indicated otherwise in the credit line; if the material is not included under the Creative Commons license, users will need to obtain permission from the license holder to reproduce the material. To view a copy of this license, visit http:// creativecommons.org/licenses/by-nc-sa/4.0/

Supplementary Information accompanies the paper on Experimental \& Molecular Medicine website (http://www.nature.com/emm) 